cholesterol level was significantly $(\mathrm{p}=0.007)$ higher among obese (192.24 \pm 41.39$)$ than non obese $(179.27 \pm 39.57)$ subjects. No significant difference of $\mathrm{Na}$ and $\mathrm{K}$ was observed between obese and non obese subjects.

Conclusion The prevalence of obesity is alarmingly high in adult population of Punjab.

\section{P1-408 PREGNANCY PLANNING AND THE ROLE OF SOCIAL INEQUALITIES IN HEALTH AND DEVELOPMENTAL OUTCOMES AT 5 YEARS}

doi:10.1136/jech.2011.142976f.98

${ }^{1} \mathrm{C}$ Carson, ${ }^{*}{ }^{2} \mathrm{~A}$ Sacker, ${ }^{1} \mathrm{M}$ Redshaw, ${ }^{2} \mathrm{Y}$ Kelly, ${ }^{1} \mathrm{~J}$ Kurinczuk, ${ }^{1} \mathrm{M}$ Quigley. ${ }^{1}$ National Perinatal Epidemiology Unit, University of Oxford, Oxford, UK; ${ }^{2}$ Institute for Social and Economic Research, University of Essex, Colchester, Essex, UK

Objective To examine the effects of unplanned pregnancy on cognitive, behavioural and health outcomes of children at 5 years.

Design Population-based cohort (Sweep 1 and 3 of the UK Millennium Cohort Study).

Participant 13529 singletons, born 2000-2001.

Exposure measure Mothers reported whether their pregnancy was planned, and their feelings when first pregnant. The population was divided into "unplanned" pregnancies (unplanned and unhappy), "mistimed" pregnancies (unplanned but happy) and "planned".

Outcome measures At 5 years mothers answered questions about their child's health and behaviour (Strength and Difficulties Questionnaire). Children completed cognitive tests (British Abilities Scales II), including a component to measure verbal abilities.

Results $17 \%$ of pregnancies were unplanned, 31\% mistimed, and $52 \%$ planned. On average, children from unplanned pregnancies had cognitive scores indicating a delay of 5 months; were more likely to have behavioural problems (OR 2.9 (95\% CI 2.3 to 3.3)); and more likely to be in poor health than the planned group (OR 2.3 (95\% CI 1.8 to 3.0)). After adjustment the effects were reduced, though remained significant for behavioural and health outcomes: OR 1.3 (95\% CI 1.1 to 1.6$)$ and OR 1.6 (95\% CI 1.2 to 2.1 ), respectively. The effects were smaller in the mistimed group, and after adjustment for confounding by socioeconomic and demographic factors, these effects disappeared.

Conclusions While the adverse outcomes associated with mistimed pregnancies can be explained by confounding of socioeconomic circumstances, there remains a significant effect on the health and behaviour of children born after unplanned pregnancies. Potential confounding by other contextual or family factors will be examined.

\section{P1-409 DIFFERENTIAL LOSS OF PARTICIPANTS DOES NOT NECESSARILY CAUSE SELECTION BIAS}

doi:10.1136/jech.2011.142976f.99

K Carter, ${ }^{*}$ F I Gunasekara, S Mckenzie, T Blakely. Health Inequalities Research Programme, Department of Public Health, University of Otago, Wellington, New Zealand

Background Most research is affected by differential participation, where individuals who do not participate have different character- istics to those who do. This is often assumed to induce selection bias. However, selection bias only occurs if the exposure-outcome association differs for participants compared to non-participants. We empirically demonstrate that selection bias does not necessarily occur when there is varying participation in a study.

Methods We used data from the first three waves of the longitudinal Survey of Family, Income and Employment (SoFIE). We examined baseline associations of labour market activity and education with self-rated health using logistic regression in four participation samples: A) the original sample at year one ( $\mathrm{N}=22265)$; $\mathrm{B})$ drop outs over 3 years $(n=3880)$; C) those remaining in the sample over 3 years $(n=18350)$; and $D)$ those (year 3 ) consenting to further data linkage $(n=14340)$.

Results Loss to follow-up was more likely among lower socioeconomic groups and those with poorer health. However, for labour market activity and education, the odds of reporting fair/poor health were similar across all samples, including after adjustment for sociodemographic variables. Thus, there was little evidence of selection bias.

Conclusions Differential loss to follow-up did not lead to selection bias in the association between socioeconomic measures and selfrated health. When assessing the possibility of selection bias researchers should consider whether differential participation affects the exposure-outcome association, not just participation by exposure or outcome separately.

\section{P1-410 EVALUATION OF INFANT MORTALITY RISK FACTORS IN AN EASTERN AREA OF IRAN; A CASE-CONTROL STUDY- 2009}

doi:10.1136/jech.2011.142976f.100

R Chaman, ${ }^{*}$ P Zolfaghari, M G Taramsari, M B Sohrabi. Shahroud University of Medical sciences, Shahroud, Iran

Introduction Infant Mortality Rate (IMR) is one of the most important indicators of community health situation and especially children health status. This study was conducted for determination of IMR risk factors in Shahroud Township (which is located in the east of Iran).

Methods This study was carried out as a Case- Control study and all infant deaths (117 cases) from 2007 to 2009 in Shahroud Township were selected. For each case two controls were entered the study by matching on time and place of birth (234 controls). After data collection of all related variables, data analysis was performed using relevant statistical methods (Univariate and multivariate conditional logistic regression).

Results Univariate analysis of risk factors showed statistical significance ( $\mathrm{P}$ Value $<0.05$ ) between infant mortality and low birth weight $(<2500 \mathrm{~g})$ with $\mathrm{OR}=15.14$, prematurity ( $<37$ weeks of gestational age) with $\mathrm{OR}=9.85$ and high risk pregnancy with $\mathrm{OR}=3.13$. Multivariate analysis resulted in significant statistical association ( $p$ value $<0.05$ ) between infant mortality and low birth weight $(\mathrm{OR}=8.04)$ and prematurity $(\mathrm{OR}=3.51)$.

Conclusion Based on our findings Low Birth Weight (LBW) and prematurity are the most important risk factors for infant mortality and prevention of these two risk factors will reduce a majority of infant mortalities. Determination of underlying causes of LBW and prematurity needs serious attention of public health investigators. 